\title{
Disruption of M1 Activity during Performance Plateau Impairs Consolidation of Motor Memories
}

\author{
Raphaël Hamel, ${ }^{1}$ Maxime Trempe, ${ }^{1,2}$ and Pierre-Michel Bernier ${ }^{1}$ \\ 'Département de kinanthropologie, Faculté des sciences de l'activité physique, Université de Sherbrooke, Sherbrooke, Québec J1K 2R1, Canada, and \\ 2Department of Sports Studies, Bishop’s University, Sherbrooke, Québec J1M 1Z7, Canada
}

\begin{abstract}
Upon exposure to a new sensorimotor relationship, motor behaviors iteratively change early in adaptation but eventually stabilize as adaptation proceeds. Behavioral work suggests that motor memory consolidation is initiated upon the attainment of asymptotic levels of performance. Separate lines of evidence point to a critical role of the primary motor cortex (M1) in consolidation. However, a causal relationship between M1 activity during asymptote and consolidation has yet to be demonstrated. The present study investigated this issue in male and female participants using single-pulse transcranial magnetic stimulation (TMS) to interfere with postmovement activity in M1 in two behavioral phases of a ramp-and-hold visuomotor adaptation paradigm. TMS was either provided after each trial of the ramp phase of adaptation when a gradual increase in the visuomotor rotation caused movements to be changing, or after each trial of the hold phase of adaptation when the rotation was held constant and movements tended to stabilize. Consolidation was assessed by measuring performance on the same task $24 \mathrm{~h}$ later. Results revealed that TMS did not influence adaptation to the new visuomotor relationship in either condition. Critically, however, TMS disruption of M1 activity selectively impaired consolidation of motor memories when it was provided during the hold phase of adaptation. This effect did not take place when TMS was delivered over adjacent dorsal premotor cortex or when motor behaviors in late adaptation were prevented from plateauing. Together, these data suggest that the impaired consolidation stemmed from interference with mechanisms of repetition-dependent plasticity in M1.
\end{abstract}

Key words: consolidation; primary motor cortex; reaching; TMS; visuomotor adaptation

\section{Significance Statement}

The present work demonstrates that TMS disruption of M1 activity impairs the consolidation of motor memories selectively when performance reaches asymptotic levels during sensorimotor adaptation. These findings provide evidence for a causal contribution of M1 to motor memory formation when movements tend to repeat, likely through mechanisms of repetition-dependent plasticity.

\section{Introduction}

Sensorimotor adaptation usually progresses through two typical phases. Upon initial exposure to a perturbation, errors drive the iterative updating of descending motor commands, causing movements to be gradually changing. Ultimately, with practice

Received Dec. 19, 2016; revised July 27, 2017; accepted Aug. 10, 2017

Author contributions: R.H., M.T., and P.-M.B. designed research; R.H. performed research; R.H., M.T., and P.-M.B. analyzed data; R.H., M.T., and P.-M.B. wrote the paper.

This work was supported by Fonds de la recherche en santé du Québec (FRSQ) and Natural Sciences and Engineering Research Council Grants, Natural Sciences and Engineering Research Council-Undergraduate Student Research Award, and Senate Research Committee of Bishop's University Research and Creative Activity Grant.

The authors declare no competing financial interests.

Correspondence should be addressed to Dr. Pierre-Michel Bernier, Faculté des sciences de l'activité physique, Université de Sherbrooke, 2500 Boulevard de I'Université, Sherbrooke, Québec J1K 2R1, Canada. E-mail: pierre-michel.bernier@usherbrooke.ca.

DOI:10.1523/JNEUROSCI.3916-16.2017

Copyright $\odot 2017$ the authors $\quad 0270-6474 / 17 / 379197-10 \$ 15.00 / 0$ and feedback, motor performance improves and eventually stabilizes at a plateau.

Once acquired, the memory representation of a novel sensorimotor relationship is kept in a labile state before it is stored into long-term memory (Shadmehr and Holcomb, 1997; Krakauer et al., 2005). This is thought to occur through a time-dependent process called "consolidation." Evidence suggests that consolidation is initiated when performance reaches asymptotic levels during adaptation (Yin and Kitazawa, 2001; Hauptmann et al., 2005; Krakauer et al., 2005; Trempe and Proteau, 2010). For instance, Yin and Kitazawa (2001) showed that 250 trials of visuomotor adaptation yielded no significant aftereffects $24 \mathrm{~h}$ later, whereas 250 additional trials of the same task led to persistent aftereffects. Given that performance had already plateaued by the 250th trial, the authors argued that additional repetitions of the stabilized behavior was critical to trigger consolidation. A similar finding was reported by Krakauer et al. (2005), whereby a newly learned visuomotor relationship became resistant to interference from a 
A

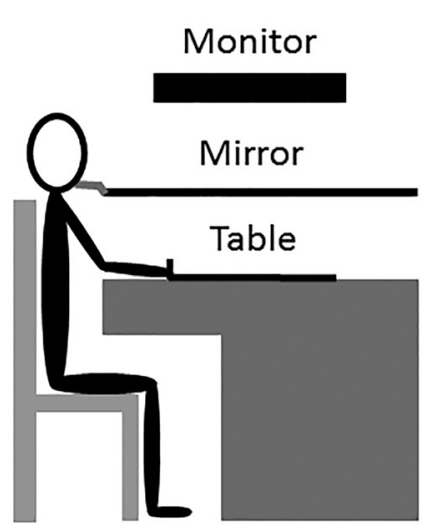

B

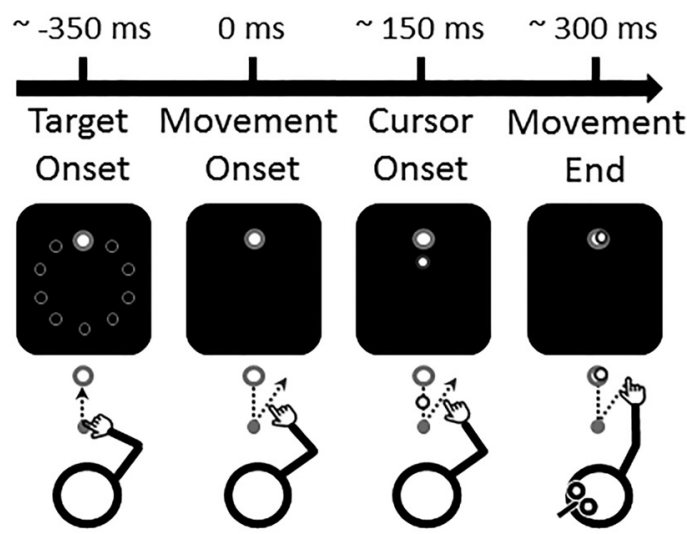

Figure 1. Schematic representation of the apparatus and experimental task. $\boldsymbol{A}$, Side view of the apparatus. $\boldsymbol{B}$, Chronology of a typical trial. Appearance of 1 of the 10 targets indicated the beginning of a trial (Target Onset). Movement onset corresponded to when the hand left the starting point (Movement Onset). Vision of the cursor was only provided once the hand crossed the halfway point between the starting point and the target (Cursor Onset). The delivery of the TMS pulse over the left M1 was triggered at movement completion (Movement End).

counter perturbation only in a condition in which an extensive period of training at asymptotic levels had occurred.

Although neural plasticity associated with sensorimotor adaptation is broadly distributed (Doyon and Benali, 2005; Lalazar and Vaadia, 2008; Shadmehr et al., 2010), converging lines of evidence point to a critical role of the primary motor cortex (M1) (Richardson et al., 2006; Hadipour-Niktarash et al., 2007; Overduin et al., 2009; Galea et al., 2011; Della-Maggiore et al., 2017). For instance, Richardson et al. (2006) used repeated transcranial magnetic stimulation (rTMS) to disrupt M1 processing before force field adaptation. They showed that rTMS led to lower performance when retested $24 \mathrm{~h}$ later, arguing that M1 would be critical for initiating the development of motor memories (for a similar finding using a finger motor skill learning task, see also Muellbacher et al., 2002). Consistent with this, HadipourNiktarash et al. (2007) applied single-pulse TMS over M1 at the end of every trial of a visuomotor adaptation protocol. They found that TMS led to a faster rate of forgetting during immediate deadaptation, suggesting a more fragile memory trace.

Although the preceding studies established a link between M1 and consolidation, they could not address whether its contribution differed between the early and late phases of adaptation. This is attributable in part to the fact that the neuromodulation techniques used (i.e., rTMS or transcranial Direct Current Stimulation) (Muellbacher et al., 2002; Richardson et al., 2006; Overduin et al., 2009; Galea et al., 2011) induce changes in cortical excitability that outlast the stimulation period and persist for extended periods of time (Hallett, 2007). Hence, their influence on M1 could not be specifically constrained to the early or the late phase of adaptation. In this regard, accumulating evidence suggests that M1 undergoes structural changes predominantly when motor performance tends to plateau (Wise et al., 1998; Paz et al., 2003, 2005; Orban de Xivry et al., 2011, 2013). For instance, M1 neurons modulate their task-related firing activity mainly in the late stage of visuomotor adaptation, possibly reflecting the initiation of motor memory consolidation (Wise et al., 1998; Paz et al., 2003, 2005). Similarly, M1 corticospinal excitability is modulated during force field adaptation only in perturbation schedules that allow movements to stabilize at a plateau, which might be key for producing M1 plasticity (Orban de Xivry et al., 2011, 2013).

Together, these results point to a greater contribution of M1 to consolidation when motor performance reaches a plateau. However, a causal relationship between consolidation and M1 activity during asymptote has yet to be demonstrated in humans. In this light, the present work assessed consolidation by measuring reaching performance $\sim 24 \mathrm{~h}$ after exposure to a novel visuomotor relationship. A ramp-and-hold adaptation paradigm was used so that movements were either iteratively changing during the ramp phase (i.e., early in acquisition) or plateauing during the hold phase (i.e., late in acquisition). To probe the causal contribution of M1 to memory consolidation in the early or the late phase of adaptation, single-pulse TMS was used to interfere with M1 processing at the end of every movement of either the ramp phase or the hold phase of adaptation. It was hypothesized that disruption of M1 activity would impair consolidation to a greater extent when applied during the hold phase compared with the ramp phase. TMS was also delivered over adjacent dorsal premotor cortex (PMd) to act as a spatial control site.

\section{Materials and Methods}

Participants. Eighty-three healthy participants $(22.1 \pm 2.7$ years, 44 females) with no self-reported neurological or psychiatric condition took part in the experiment. They were all self-declared right-handed with normal or corrected-to-normal vision. Participants provided written informed consent for their participation in the study. They were naive as to the purpose of the experiment and had no prior experience with the task. All received a monetary compensation of $\$ 20$ (Canadian) for their participation in the study. Experimental procedures were approved by the ethical committee of the Centre Hospitalier de l'Université de Sherbrooke. One participant was excluded from all analyses for not following the experimental procedures.

Apparatus. The experimental setup consisted of a table supporting a computer monitor, which projected visual stimuli on a mirror positioned horizontally in front of participants (Fig. 1A). The monitor (20 inch Dell P1130; resolution: $1024 \times 768$; refresh rate: $150 \mathrm{~Hz}$ ) was mounted face down $29 \mathrm{~cm}$ above the horizontal mirror, and the mirror was mounted $29 \mathrm{~cm}$ above the table. Thus, the visual stimuli appeared to be projected directly onto the surface of the table on the same plane as the hand. Because of the mirror, participants could not see their hand. A 2-joint planar manipulandum was placed on the table and was held by participants via a stylus located at its mobile end. The manipulandum was custom-built with two lightweight metal rods ( 48 and $45 \mathrm{~cm}$ for the distal and proximal rods, respectively), with the fixed end attached to the upper left corner of the table. A thin sheet of smooth plastic covered the table surface, and foam pads were installed under the hinges, allowing the manipulandum to be moved everywhere on the table with minimal inertia and friction.

Two potentiometers positioned in the joints of the manipulandum allowed the measurement of the angle of each segment at $1000 \mathrm{~Hz}$ from 


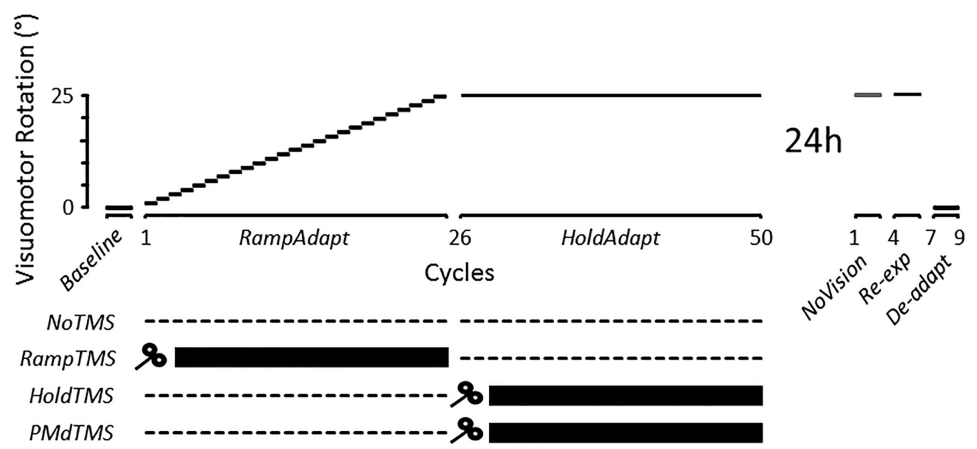

Figure 2. Time course of the main experimental protocol. On separate days ( $24 \mathrm{~h}$ apart), participants performed an acquisition and a retention session of a visuomotor adaptation task. In RampAdapt, a CCW visuomotor rotation was gradually introduced at the rate of $1^{\circ}$ per cycle from $1^{\circ}$ to $25^{\circ}$ over 25 cycles ( 250 trials). In HoldAdapt, the rotation was held at $25^{\circ} \mathrm{CCW}$ for 25 additional cycles (250 trials). Participants were separated into three groups and received (or not) TMS pulses in different phases of the acquisition session. Participants of the NoTMS group ( $n=14)$ did not receive TMS during acquisition. Participants of the RampTMS $(n=14)$ and HoldTMS groups $(n=13)$ received single-pulse TMS over M1 after each trial of either the RampAdapt or HoldAdapt phase, respectively. Participants of the PMdTMS group $(n=14)$ received single-pulse TMS over the PMd after each trial of the HoldAdapt phase. The retention session was used to assess consolidation. The NoVision phase was performed without visual feedback of the hand. The Re-exp phase consisted of the reexposure to the $25^{\circ} \mathrm{CCW}$ rotation, whereas the De-adapt phase was performed with veridical (i.e., nonrotated) visual feedback of the hand.

which the $2 \mathrm{D}$ position of the stylus was calculated. Raw kinematic data were spatially smoothed with a Kalman filter to estimate hand position in real time. This information was then used to project a cursor corresponding to the participants' hand. The time lag between the measurement of the angles and the projection of the cursor was between 7 and $9 \mathrm{~ms}$, as determined in separate pilot experiment using a high-speed camera $(1000 \mathrm{~Hz})$.

Procedures. Participants had to perform center-out reaching movements with their right hand toward 1 of 10 visual targets (Fig. 1B). All targets consisted of green circles of $0.5 \mathrm{~cm}$ radius. They were positioned along a circular array of $10 \mathrm{~cm}$ radius. At the center of the workspace, a gray circle of $0.75 \mathrm{~cm}$ radius served as the starting point for every trial. It was located $30 \mathrm{~cm}$ in front of the participants' chest along the midline. The cursor representing the hand position consisted of a red circle of $0.29 \mathrm{~cm}$ radius.

To initiate a trial, participants had to bring the cursor into the starting point and remain stationary within its boundary for $1 \mathrm{~s}$. This prompted the disappearance of the starting point, which indicated the beginning of the trial. After $1.5 \mathrm{~s}$, a target was presented, instructing participants to initiate their reaching movement. Target appearance was pseudorandomized so that each target was presented once every 10 trials (i.e., cycle). Participants were asked to produce straight movements with minimal online corrections in a targeted movement time of $300 \mathrm{~ms}$. This ensured that all participants had a similar speed-accuracy trade-off (Fitts, 1954). Movement end corresponded to when the tangential velocity of the cursor dropped to $<0.05 \mathrm{~cm} / \mathrm{s}$. Vision of the cursor was provided only during the second half of the movement. Specifically, the cursor appeared once the hand crossed an imaginary $5 \mathrm{~cm}$ radius from the starting point. This corresponded to $\sim 150 \mathrm{~ms}$ into the movement, and thus $\sim 150$ ms before movement end.

The target and final cursor positions remained displayed on the screen for $500 \mathrm{~ms}$ after movement completion, after which they disappeared. To limit exposure to the visuomotor rotation during the return to the starting point, the cursor was only provided when it was within an imaginary $1.79 \mathrm{~cm}$ radius around the starting point. On average, $5 \mathrm{~s}$ separated each trial.

All participants took part in an acquisition session and a retention session performed on separate days and separated by $\sim 24 \mathrm{~h}$ (Fig. 2). The acquisition session began with a familiarization phase allowing participants to learn the spatial and temporal requirements of the task. It consisted of nine cycles ( 90 trials) in which the mapping between the hand and the cursor was veridical. Then, participants performed 50 cycles (500 trials) over the course of which a visuomotor rotation was introduced between the hand and its corresponding cursor. Over cycles 1-25 (250 trials), a counterclockwise (CCW) visuomotor rotation was gradually introduced at a rate of $1^{\circ}$ every cycle, up to $25^{\circ}$ (hereafter called the "RampAdapt" phase). Then, over cycles $26-50$ (250 trials), the visuomotor rotation remained constant at $25^{\circ}$ (hereafter called the "HoldAdapt" phase). One minute breaks were provided every 10 cycles to allow participants to rest. Upon completion of the acquisition session, participants were told to resume their daily activities and were invited to come back the next day for the retention session. Importantly, participants were not informed that a visuomotor rotation had been introduced, and none of them became consciously aware of it during the acquisition session, thus excluding the possibly that conscious strategies influenced the results.

Consolidation was assessed in the retention session, using three different tests that have been used in the literature. More specifically, participants performed 3 cycles ( 30 trials) with no visual feedback of the cursor and no endpoint feedback (hereafter called the "NoVision" phase). The persistence of the adapted behavior in absence of corrective visual feedback is thought to reflect the retention/consolidation of motor memory (Galea et al., 2015). Then, participants performed 3 cycles (30 trials) in which they were reexposed to the $25^{\circ}$ visuomotor rotation (hereafter called the "Re-exp" phase). Participants' capacity to reacquire the newly learned visuomotor relationship (i.e., savings) is also a reflection of the strength of a motor memory (Smith et al., 2006; Herzfeld et al., 2014). Finally, participants performed 3 cycles ( 30 trials) in which the mapping between the hand and the cursor was made veridical again (i.e., $0^{\circ}$ visuomotor rotation; hereafter called the "De-adapt" phase). The presence of aftereffects in this context is yet another behavioral evidence of motor memory retention/consolidation (Hadipour-Niktarash et al., 2007; Trempe and Proteau, 2010; Landi et al., 2011). It was reasoned that, if TMS interfered with consolidation of motor memories, it should lead to differences in each of these tests of retention/consolidation. The main statistical analysis thus exploited data from all of these phases to obtain the most comprehensive assessment of consolidation. Nevertheless, as a confirmatory analysis, performance was also compared across groups using only data from the NoVision phase, which can be taken as the most direct reflection of consolidation without any possible influence of being reexposed to the visuomotor rotation.

TMS and EMG. To assess the causal role of M1 in the consolidation of motor memories, single-pulse TMS was used to interfere with contralateral (left) M1 activity in either one of two phases: (1) when movements were gradually changing (RampAdapt phase); or (2) when movements were stabilizing (HoldAdapt phase). This was done by creating two separate groups, which differed with respect to the phase of the acquisition session in which TMS was delivered. Participants of the RampTMS group $(n=14)$ received single-pulse TMS over M1 at the end of each movement of the RampAdapt phase (i.e., cycles 1-25). Participants of the HoldTMS group ( $n=13$ ) received single-pulse TMS over M1 at the end of each movement of the HoldAdapt phase (i.e., cycles 26-50). Acting as a control, a third group (NoTMS; $n=14$ ) received no TMS during the acquisition session. Finally, a fourth group was used to act as a sham and to provide spatial specificity to the results obtained at M1. Specifically, participants of the HoldPMd group $(n=14)$ received single-pulse TMS over the contralateral (left) PMd at the end of each movement of the HoldAdapt phase. This controlled for the possibility that the TMS effect hypothesized to be observed over M1 might be attributable to current spread from M1 to PMd (Hadipour-Niktarash et al., 2007). This group also controlled for a possible effect of distraction produced by the stimulator during the critical HoldAdapt phase.

A MagStim 200 monophasic stimulator (MagStim) with a 70-mmdiameter figure-of- 8 coil was used. The coil was placed tangentially to the 
scalp with the handle pointing backwards at a $45^{\circ}$ angle relative to the anteroposterior axis. Coil placement was determined by recording motor evoked potentials (MEPs) of the right first dorsal interosseous (FDI) muscle using surface EMG. The skin was first cleaned with alcohol swabs saturated with $70 \%$ isopropyl alcohol to reduce electrode impedance. The reference electrode was placed on the lateral epicondyle of the right humerus. Before the beginning of the acquisition session, single-pulse TMS was delivered to the left M1 to localize the FDI motor "hot spot" (i.e., the site where maximal MEPs were elicited in the FDI at $50 \pm 5 \%$ of the maximum stimulator output). The resting motor threshold at the motor hot spot was then defined as the minimum intensity required to elicit at least $5 \mathrm{MEPs}$ of 10 consecu-

tive attempts in the FDI muscle. During the experiment, single-pulse TMS was delivered at $120 \%$ resting motor threshold over the left FDI hot spot. The experimenter holding the TMS coil continuously monitored the MEPs in real-time via a computer monitor, ensuring correct positioning of the coil. Although EMG was only recorded for the FDI during the experiment, confirmatory tests were conducted to confirm that the TMS intensity and location used in the experiment also generated potent MEPs in the biceps muscle, a more proximal agonist in the present reaching task. In addition, using similar TMS parameters as used here, Schulze-Bonhage et al. (1998) demonstrated considerable overlap between the cortical areas from which MEPs could be evoked in the FDI and the deltoid, the latter also being recruited in the present task. In this light, the current stimulation site over the FDI hot spot most likely also influenced proximal arm representations used for reaching.

For stimulation of the left PMd, the coil was positioned $2 \mathrm{~cm}$ rostral and $1 \mathrm{~cm}$ medial from the left FDI hot spot (Hadipour-Niktarash et al., 2007). This location was based on neuroimaging work demonstrating that PMd is located $\sim 1.5-2.5 \mathrm{~cm}$ anterior to the hand area of the motor cortex (Fink et al., 1997; Picard and Strick, 2001). To simulate a coactivation of PMd induced by current spread from TMS over M1, the stimulation intensity over the PMd was reduced to correspond to $\sim 90 \%$ of the intensity used over M1 (Gerschlager et al., 2001; Hadipour-Niktarash et al., 2007). This incurred small, but discernable, MEPs in most participants. Across all participants, mean TMS output power was $52 \pm 3 \%$ and $46 \pm 4 \%$ over M1 and PMd, respectively.

The delivery of the TMS pulse was time-locked to the end of the movement (i.e., cursor velocity dropping to $<0.05 \mathrm{~cm} / \mathrm{s}$ ). This particular timing was chosen because response-specific processing in motor areas has been shown to begin at $\sim 150 \mathrm{~ms}$ after the onset of a visual stimulus (Ledberg et al., 2007) and has been shown to contribute to short-term retention (Hadipour-Niktarash et al., 2007). Therefore, the delivery of TMS at movement end corresponded to $\sim 150 \mathrm{~ms}$ after the hand cursor was provided. Importantly, TMS could not disrupt movement kinematics of an ongoing movement because it was provided after its completion (Orban de Xivry et al., 2011).

Data reduction. A custom-designed MATLAB script (version R2014a; MathWorks) was used to display and acquire kinematics and EMG data during the experiment. The cursor position data for each movement was acquired at $1000 \mathrm{~Hz}$ and normalized over the movement time period $(0 \%-100 \%)$.

To assess whether TMS affected movement kinematics, we first calculated participants' reaction time (RT, i.e., the time between target onset and movement onset), movement time (MT, i.e., the time between movement onset and movement end), and time to peak tangential velocity (TtPV, i.e., the time between movement onset and PV). In addition, we calculated the time between cursor onset and the TMS pulse (TCO-TMS) for participants in the RampTMS, HoldTMS, and PMdTMS groups.

Trials were excluded from all analyses if RT or MT were \pm 3 SDs around each participant's mean or if the absolute distance between the target and cursor endpoint was $>10 \mathrm{~cm}$. Overall, $3 \%$ of the trials were rejected.
Adaptation to the visuomotor rotation was assessed by measuring the hand direction at peak tangential velocity (PV). It was calculated as the angular difference between the reference vector joining the starting point and the target and the vector joining the starting base and the hand at PV. This early kinematic marker (mean $113 \mathrm{~ms}$ after movement onset; see Results) was chosen because it is considered a reflection of the movement planning process (Carlton, 1992).

The extent to which reach directions changed across trials (hereby called "directional change") in each experimental phase was calculated by computing for each participant the slope of a linear regression using the hand direction at PV data over trials 1-250 (RampAdapt phase) and trials 251-500 (HoldAdapt phase), separately.

Success at achieving the target (hereby called "hit rate") was assessed by calculating the percentage of trials in which the cursor was in contact with the target at the end of the movement (i.e., the distance between the center of the cursor and the center of the target was below the sum of their radii, $0.79 \mathrm{~cm}$ ). This was computed over trials 1-250 (RampAdapt phase) and trials 251-500 (HoldAdapt phase), separately.

Statistical analyses. The first analysis sought to assess whether TMS influenced reach kinematics and adaptation to the new visuomotor relationship during acquisition. This was done by submitting the RT, MT, $\mathrm{TtPV}$, hand direction at PV, directional change, and hit rate data to separate 4 Groups (NoTMS, RampTMS, HoldTMS, PMdTMS) $\times 2$ Phases (RampAdapt, HoldAdapt) mixed-effects ANOVAs. To ensure the TMS pulses were delivered at the same time across groups, the TCO-TMS data were submitted to a 3 groups one-way ANOVA comparing the RampTMS group (using data from the RampAdapt phase, i.e., when these participants received TMS), the HoldTMS group (using data from the HoldAdapt phase), and the PMdTMS group (using data from the HoldAdapt phase).

The second analysis tested whether TMS influenced consolidation of the new visuomotor relationship. This was done by submitting the hand direction at PV data to a 4 Groups (NoTMS, RampTMS, HoldTMS, PMdTMS) $\times 3$ Phases (NoVision, Re-exp, De-adapt) mixed-effects ANCOVA using the mean hand direction at PV over the last 30 trials of the HoldAdapt phase as a covariate. As a confirmatory analysis, the ANCOVA was also run using only data from the NoVision phase. Data are reported as mean \pm SEM.

\section{Results}

To determine whether our experimental manipulation succeeded in creating two distinct phases (i.e., one in which movements were constantly changing and one in which movements stabilized), we compared the directional change in each phase. The analysis confirmed that mean directional change was greater in the RampAdapt phase $(0.083 \pm 0.001 \%$ trial $)$ than in the HoldAdapt phase $\left(0.008 \pm 0.001^{\circ} /\right.$ trial $)$, as revealed by a significant main effect of Phase $\left(F_{(1,51)}=2884.3, p<0.001, \eta_{p}^{2}=0.98\right.$; see Fig. $3 A, B)\left({ }^{*}\right.$ denotes $\left.p<0.05\right)$. There was neither a main effect of Group $(p=0.65)$ nor an interaction $(p=0.32)$. 

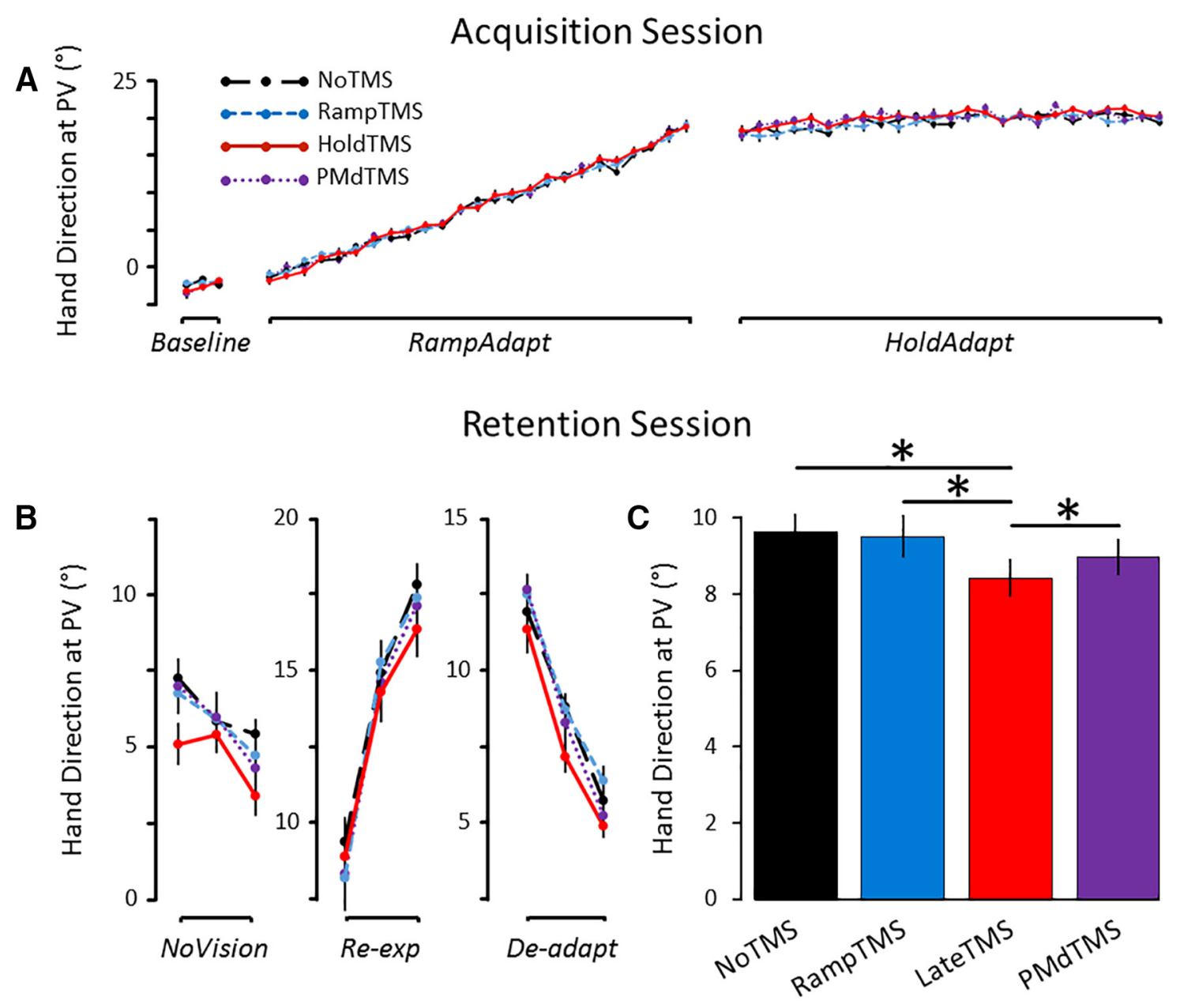

Figure 4. Main experiment results. $\boldsymbol{A}$, Mean hand direction at PV in each cycle of the acquisition session. The four groups did not differ in the extent of adaptation to the visuomotor rotation $(p=0.87)$. $\boldsymbol{B}$, Mean hand direction at PV in each cycle of the retention session. $\boldsymbol{C}$, Mean hand direction at PV of all trials performed in the retention session. The HoldTMS group showed impaired consolidation compared with each of the other three groups $\left({ }^{*} p<0.05 \mathrm{Holm}\right.$-Bonferroni corrected). Error bars indicate SEM.

The first series of analyses sought to assess whether TMS influenced reach kinematics and adaptation to the new visuomotor relationship during the acquisition session. As can be seen in Figure $4 A$, the four groups showed a very similar pattern of adaptation during the acquisition session. Namely, the hand direction at $\mathrm{PV}$ gradually changed from $\sim-2^{\circ}$ to $\sim 19^{\circ}$ in the RampAdapt phase and stabilized at $\sim 21^{\circ}$ in the HoldAdapt phase. This is supported by the ANOVA conducted on hand direction at $\mathrm{PV}$, which revealed a significant main effect of Phase $\left(F_{(1,51)}=11741, p<0.001, \eta_{p}^{2}=0.99\right)$, with hand direction at PV being more shifted to the right in the HoldAdapt phase $\left(20.3 \pm 0.2^{\circ}\right)$ compared with the RampAdapt phase $\left(8.0 \pm 0.2^{\circ}\right)$. Most importantly, however, the ANOVA revealed no significant main effect of Group $(p=0.87)$ and no interaction $(p=0.28)$, suggesting that the four groups adapted to the new visuomotor relationship to the same extent during acquisition.

Separate ANOVAs conducted on RT, MT, and TtPV were used to assess a potential influence of TMS on movement kinematics. Results revealed neither a significant main effect nor an interaction for RT $(367 \pm 3 \mathrm{~ms})$ and TtPV $(113 \pm 1 \mathrm{~ms})$. However, there was a significant interaction for $\operatorname{MT}\left(F_{(3,51)}=2.9, p<\right.$ $\left.0.04, \eta_{p}^{2}=0.15 ; 315 \pm 3 \mathrm{~ms}\right)$. Breakdown of the interaction revealed that MT during the Ramp phase was longer for the NoTMS group $(337 \pm 9 \mathrm{~ms})$ than for the RampTMS group
(300 $\pm 9 \mathrm{~ms})$. Importantly, there was no significant difference in TCO-TMS between groups ( $166 \pm 2 \mathrm{~ms})$.

As for task success, the ANOVA performed on the hit rate data revealed a significant main effect of Phase $\left(F_{(1,51)}=48.4, p<\right.$ $\left.0.001, \eta_{p}^{2}=0.49\right)$, suggesting that participants were significantly more accurate in the HoldAdapt phase compared with the RampAdapt phase $(46 \pm 2 \%$ and $38 \pm 2 \%$, respectively). However, there was neither a main effect of Group $(p=0.38)$ nor an interaction $(p=0.48)$.

Overall, these results suggest that postmovement TMS provided either in the RampAdapt or the HoldAdapt phase did not disrupt adaptation to the new visuomotor relationship or the movement kinematics and success rates.

The second analysis sought to assess whether TMS provided over M1 in the RampAdapt phase or over M1 or PMd during the HoldAdapt phase influenced the consolidation of the new visuomotor relationship $24 \mathrm{~h}$ later. The hand direction at PV data across each cycle of the retention session is presented in Figure $4 B$. As can be seen, participants expressed approximately onethird of the adapted behavior in the NoVision condition, with hand directions at PV at $\sim 6^{\circ}$. They then showed a rapid readaptation upon reexposure to the rotation and the reverse effect upon removal of the rotation in the deadaptation phase. Most importantly, consistent with the hypothesis, the HoldTMS group 
showed impaired retention over all three phases compared with the RampTMS, NoTMS, and PMdTMS groups (Fig. 4C). This was confirmed by the ANCOVA, which revealed a significant main effect of Group $\left(F_{(3,50)}=3.12, p=0.03, \eta_{p}^{2}=0.16\right)$. There was neither a main effect of Phase $(p=0.5)$ nor an interaction $(p=0.9)$. Holm-Bonferroni corrected pairwise comparisons revealed that hand direction at PV for the HoldTMS group (mean across the three phases of $8.4 \pm 0.5^{\circ}$ ) was significantly lower than for the NoTMS group $\left(9.6 \pm 0.5^{\circ} ; p=0.02\right)$, the RampTMS group $\left(9.5 \pm 0.6^{\circ} ; p=0.03\right)$, and the PMdTMS group (9.4 $\pm 0.5^{\circ}$; $p=0.04)$. No significant difference was observed between the NoTMS, RampTMS, and PMdTMS groups (all $p$ values $>0.6$ ).

As a confirmatory analysis, the ANCOVA was also performed using only data from the NoVision phase. The pattern of results was the same, with a main effect of Group $\left(F_{(3,50)}=2.79, p=\right.$ $\left.0.049, \eta_{p}^{2}=0.14\right)$, and Holm-Bonferroni corrected pairwise comparisons revealing that the HoldTMS group presented significantly impaired consolidation compared with each of the other three groups (all $p$ values $<0.05$ ).

\section{Control experiment}

Results from the main experiment suggest that TMS interfered with the consolidation of motor memories in M1 specifically when performance was plateauing in the HoldAdapt phase. However, an inherent feature of the present protocol is that TMS was delivered over M1 later in the acquisition session for the HoldTMS group than the RampTMS group. Hence, it may be that TMS disrupted consolidation not because it was delivered when performance was plateauing, but because it was delivered at the end of the acquisition session. To test that, two additional groups were tested in a control experiment. They were submitted to a perturbation schedule in which visuomotor rotations kept changing both early (i.e., cycles 1-25; RampAdapt phase) and late (i.e., cycles 26-50; VarAdapt phase) in the acquisition session, thereby preventing performance from plateauing (see Fig. 5A). Specifically, during the VarAdapt phase, visuomotor rotations gradually increased to $31^{\circ}$ (i.e., cycle 32 ), then decreased to $19^{\circ}$ (i.e., cycle 44), then increased back to $25^{\circ}$ (i.e., cycle 50) at the rate of $\pm 1^{\circ}$ per 10 trials.

A first group ("VarTMS"; $n=14$ ) received single-pulse TMS over M1 at the end of each trial of the VarAdapt phase. It was compared with a second group ("VarNoTMS"; $n=14$ ), which did not receive TMS and thus acted as a control. The hypothesis that TMS over M1 interfered with consolidation specifically because of the performance plateau would be supported if consolidation did not differ between the VarTMS and VarNoTMS groups. This is because TMS would be delivered over M1 in a context in which motor behaviors did not plateau.

The same dependent variables were used as in the main experiment, with the exception of the calculation of directional change during the VarAdapt phase. Specifically, directional change was assessed by averaging the absolute values of the slopes of three linear regressions fitted over trials 251-320, 321-440, and 441500. This was done to capture the perturbation schedule of the VarAdapt phase. A paired $t$ test comparing the slopes of all participants from the main experiment during the Hold phase and all participants from the control experiment during the VarAdapt phase confirmed that movements were indeed more continuously changing in the control experiment $\left(0.063 \pm 0.014^{\circ} /\right.$ trial $)$ compared with the main experiment $\left(0.008 \pm 0.006^{\circ} /\right.$ trial $)$ $\left(t_{(81)}=25.0, p<0.001 ; d=6.7\right)$.

\section{Results}

As can be visually appreciated from Figure $5 B$, the manipulation of the perturbation schedule was successful in preventing a performance plateau, with motor behaviors continuously changing throughout the course of the acquisition session.

Analysis of the hand direction at PV revealed that the VarTMS and VarNoTMS groups adapted to the new visuomotor relationship to a similar extent. Indeed, the ANOVA revealed only a main effect of Phase $\left(F_{(1,26)}=6198.0, p<0.001, \eta_{p}^{2}=0.99\right)$, with hand direction at PV being significantly more shifted to the right in the VarAdapt phase $\left(20.9 \pm 0.3^{\circ}\right)$ compared with the RampAdapt phase $\left(8.1 \pm 0.2^{\circ}\right)$. However, the ANOVA revealed neither a significant main effect of Group $(p=0.28)$ nor an interaction $(p=0.22)$. Among the other dependent variables (RT, MT, TtPV, TCO-TMS, hit rate), only RT presented a significant main effect of Group during the acquisition session, with the VarTMS group initiating their movements slightly faster than the VarNoTMS group ( $381 \pm 10 \mathrm{~ms}$ and $412 \pm 10 \mathrm{~ms}$, respectively; $p<0.05)$. TCO-TMS in the VarTMS group was $172 \pm 4 \mathrm{~ms}$.

Most importantly, as can be seen in Figure $5 C, D$, there was no significant difference in consolidation between the VarNoTMS and VarTMS groups $\left(9.8 \pm 0.4^{\circ}\right.$ and $9.9 \pm 0.4^{\circ}$, respectively). This was confirmed by the ANCOVA, which revealed no significant main effect of Group $\left(F_{(1,25)}=0.02, p=0.88, \eta_{p}^{2}=0.001\right)$, no main effect of Phase $(p=0.47)$, and no interaction $(p=0.28)$. There was also no significant difference across groups when performing the ANCOVA using only trials from the NoVision phase $(p=0.36)$.

Overall, the control experiment confirmed that the impaired retention presented by the HoldTMS group in the main experiment was specifically attributable to the fact that TMS was applied during a performance plateau and not simply because it was delivered late in the acquisition session.

\section{Discussion}

In this study, the contribution of M1 to the consolidation of motor memories was investigated in two characteristic behavioral phases of a ramp-and-hold visuomotor adaptation paradigm. Results revealed that TMS did not influence adaptation to the new visuomotor relationship during acquisition, but selectively impaired consolidation when it was provided during the hold phase of adaptation. This effect was specific to M1 as it was not observed when stimulating the PMd. A control experiment further confirmed the critical role of behavioral plateauing because TMS did not impair consolidation when performance late in acquisition was prevented from plateauing. These findings extend a series of studies that have used neuromodulation either before (Richardson et al., 2006) or during adaptation (see also Muellbacher et al., 2002; Hadipour-Niktarash et al., 2007; Overduin et al., 2009; Galea et al., 2011) to probe the contribution of M1 to motor memory formation. Whereas these studies all pointed to a role of M1 in consolidation, the present results are the first to specifically attribute the contribution of M1 to the attainment of a performance plateau during visuomotor adaptation. In doing so, they provide causal evidence that processes associated with consolidation are engaged in M1 when performance reaches asymptotic levels.

The present protocol successfully allowed us to manipulate the degree to which movements were repeated across different phases of adaptation, with reach trajectories being more consistent during HoldAdapt compared with RampAdapt. In this light, the most likely possibility accounting for the present results is that TMS interfered with mechanisms of repetition-dependent 


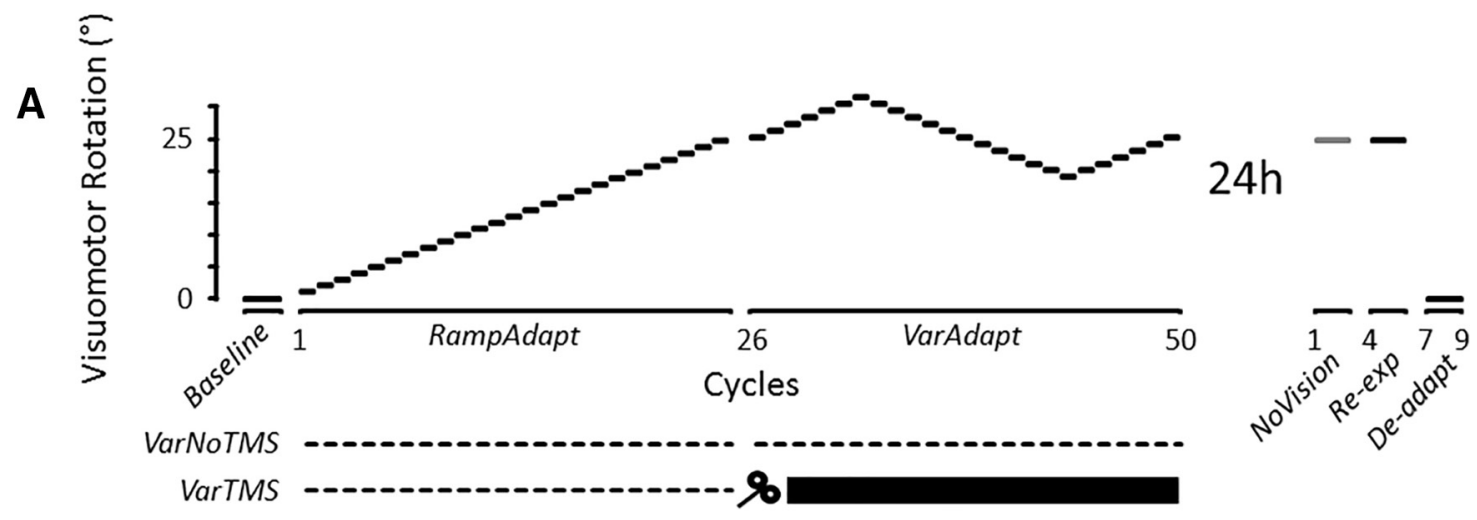

\section{Acquisition Session}

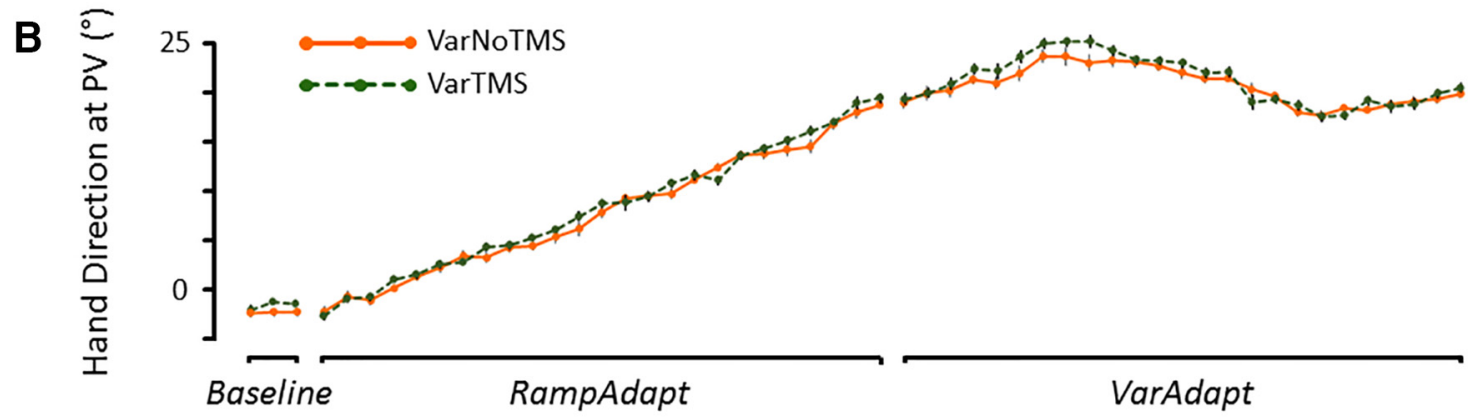

\section{Retention Session}
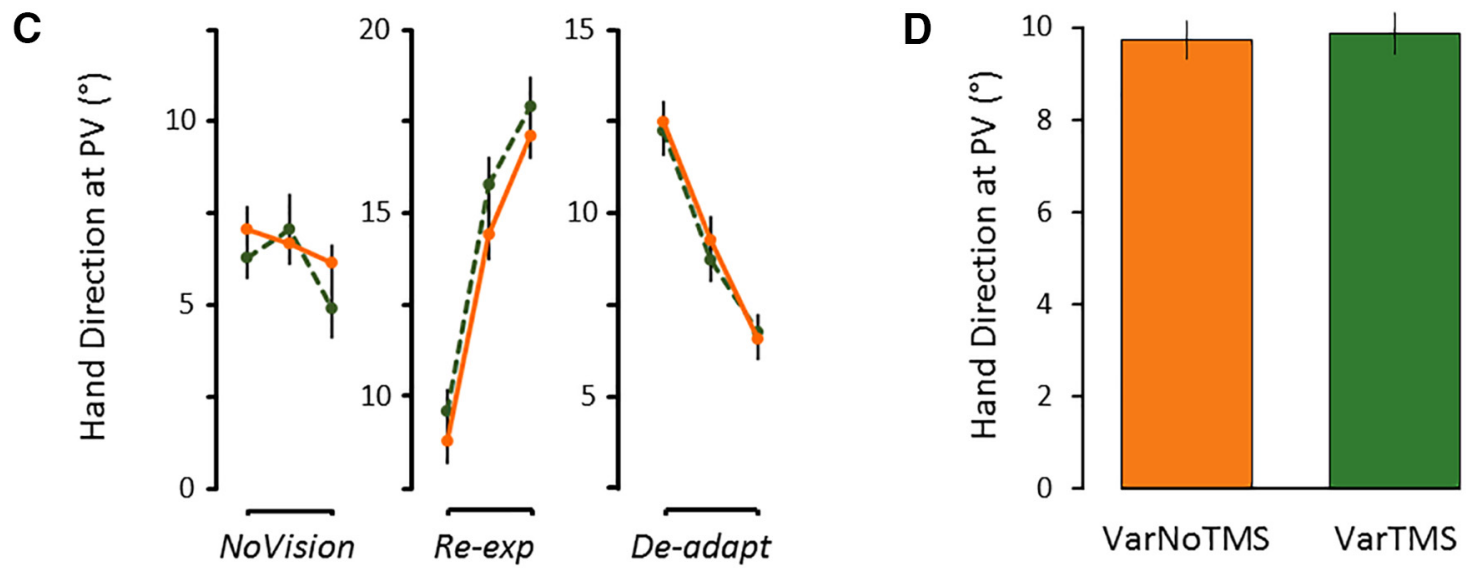

Figure 5. Control experiment. $\boldsymbol{A}$, Two additional groups were tested in a schedule in which visuomotor rotations kept iteratively changing both early and late in the acquisition session. Participants of the VarNoTMS group $(n=14)$ did not receive TMS, whereas those of the VarTMS group $(n=14)$ received TMS after each trial of the VarAdapt phase. $\boldsymbol{B}$, Mean hand direction at PV in each cycle of the acquisition session. The VarNoTMS and VarTMS groups did not differ in the extent of adaptation to the visuomotor rotation $(p=0.28)$. C, Mean hand direction at PV in each cycle of the retention session. $D$, Mean hand direction at PV of all trials performed in the retention session. The VarNoTMS and VarTMS groups did not differ from each other $(p=0.88)$. Error bars indicate SEM.

plasticity in M1 (also called Use-Dependent Plasticity [UDP] in recent literature). Indeed, the repetition of movements is thought to strengthen existing neural connections and facilitate the creation of new ones within M1 through LTP-like mechanisms (Pascual-Leone et al., 1994; Bütefisch et al., 2000). Although repetition-dependent plasticity has been well documented for simple finger movements (Classen et al., 1998; Bütefisch et al., $2000,2004)$, it has also recently been extended to more complex upper-limb reaching movements and proposed to contribute to sensorimotor adaptation (Diedrichsen et al., 2010; Huang et al.,
2011; Verstynen and Sabes, 2011; McDougle et al., 2015). Specifically, the tight distribution of movement trajectories associated with a performance plateau would constitute a key step for the induction of UDP (Huang et al., 2011), biasing reach trajectories toward those converged upon during adaptation (Diedrichsen et al., 2010; Huang et al., 2011; Orban de Xivry et al., 2011, 2013; Verstynen and Sabes, 2011; Leow et al., 2014, 2016). In support, Leow et al. (2014) used anodal transcranial Direct Current Stimulation over M1, a technique known to facilitate synaptic plasticity and increase UDP, whereas participants adapted to a new 
visuomotor relationship. They found that it significantly impaired adaptation to a second distinct rotation (i.e., anterograde interference) but only when the first rotation had been practiced extensively at asymptotic levels. In this light, TMS provided at movement end may have disrupted the direction-dependent memory trace that forms in $\mathrm{M} 1$ upon repeated movements (Classen et al., 1998), thus weakening the consolidation of the adapted reach trajectories.

Although repetition-dependent plasticity is likely to account for the present results, several considerations should be raised. First, studies investigating UDP in the context of sensorimotor adaptation have used paradigms involving few targets restricted to a narrow area of space (Diedrichsen et al., 2010; Huang et al., 2011; Verstynen and Sabes, 2011; McDougle et al., 2015), unlike the multiple target directions used here. Although it is reasonable to conjecture that the UDP mechanisms identified in these previous protocols would also take place for more numerous targets, the degree to which UDP contributes to sensorimotor adaptation when movements in a given direction repeat at a lesser rate remains unknown. Second, although UDP has been shown to exert a transient bias on reach trajectories (Diedrichsen et al., 2010; Huang et al., 2011; Verstynen and Sabes, 2011; McDougle et al., 2015), to our knowledge, its influence on the long-term (i.e., $24 \mathrm{~h}$ ) retention of motor memories has not been specifically tested. Still, there is evidence that changes in the directional tuning of M1 neurons, which occur primarily during performance asymptote (Paz et al., 2003), persist across test sessions spanning several days (Mandelblat-Cerf et al., 2011; Richardson et al., 2012). This provides indirect evidence in favor of a contribution of repetition-dependent mechanisms in $\mathrm{M} 1$ to long-term memory consolidation. Third, adaptation is sensitive to the type of perturbation schedule, with abrupt and gradual schedules having different contributions of error-based, strategic, and repetitiondependent mechanisms (Huang et al., 2011; Taylor et al., 2014; Orban de Xivry and Lefèvre, 2015). Although the neural correlates of adaptation to abrupt and gradual perturbations schedules may differ (Muellbacher et al., 2001; Schlerf et al., 2012; Werner et al., 2014), both have been found to produce a stabilized motor memory (Hadipour-Niktarash et al., 2007; Galea et al., 2011). Furthermore, abrupt schedules typically lead to more trials spent at asymptote, which has been shown to favor UDP mechanisms in M1 (Orban de Xivry et al., 2011; Orban de Xivry and Lefèvre, 2015). Hence, it is likely that the results observed here would generalize to contexts in which a performance asymptote is reached following an abrupt perturbation. Finally, there was no influence of TMS on adjacent PMd (see also Hadipour-Niktarash et al., 2007), although neurons in this region show changes in directional tuning during force field adaptation (Xiao et al., 2006). Given that M1 is certainly not the sole contributor to consolidation (Herzfeld et al., 2014), it will be interesting to investigate repetition-dependent mechanisms in higher-order regions outside of $\mathrm{M} 1$.

It is important to note that the presence of a performance asymptote often correlates with an increased rate of task success. Indeed, as performance improves and movements become more repetitive, participants also tend to receive more frequent rewards (implicit or explicit). This was the case in the present work, as the HoldAdapt phase was associated with higher task success (i.e., hit rates) compared with the RampAdapt phase. Interestingly, Huang et al. (2011) showed that UDP-induced reach biases are larger when movement repetitions take place within an adaptation paradigm in which movements are directed toward a goal compared with repetitions alone (Verstynen and Sabes, 2011).
They argued that repetitions in the context of reducing errors may itself constitute a reward signal that would modulate the efficacy of UDP. Similarly, recent studies using finger skill tasks have revealed changes in M1 excitability (Bagce et al., 2013) and increased UDP in M1 (Mawase et al., 2017) only in groups who successfully learned the skill compared with groups that made comparable reaching actions without accumulating learning. In this context, a likely possibility is that repetition-dependent plasticity in the present experiment may have been potentiated by rewards associated with task success during asymptote.

Although the processes underlying consolidation may partly differ between sensorimotor adaptation and the learning of new motor skills (Baraduc et al., 2004; Doyon et al., 2009), the present results are consistent with findings stemming from the motor skill learning literature. Indeed, there is considerable evidence that late stages of learning are associated with increased M1 reorganization (Ungerleider, 2002; Rosenkranz et al., 2007; Masamizu et al., 2014). Importantly, the repetition of movements seems to be the key factor triggering consolidation and M1 reorganization (Karni et al., 1995, 1998; Nudo et al., 1996; Kantak et al., 2010; Gabitov et al., 2014, 2015; Reis et al., 2015; Rroji et al., 2015). For instance, Kantak et al. (2010) showed that rTMS applied over M1 before training caused an impairment in retention under a constant practice structure but not under a variable practice structure. Similarly, Gabitov et al. (2014) trained participants on a fingerto-thumb opposition task while recording fMRI and found that M1 activity upon task repetition constituted a reliable neural signature for motor memory consolidation. Hence, the present work bridges a gap between the sensorimotor adaptation and motor skill learning literature by showing that movements performed repeatedly during asymptote trigger important synaptic changes in M1.

At the cellular level, there is accumulating evidence that repeated motor experience promotes synaptogenesis and induces functional map reorganization within M1 that directly underlie motor memory formation (Kleim et al., 2004; Manto et al., 2006; Fu and Zuo, 2011; Yu and Zuo, 2011; Fu et al., 2012; Rogerson et al., 2014). Indeed, studies in rodents have demonstrated that motor skill learning leads to the formation and clustering of new dendritic spines in M1 pyramidal neurons (Xu et al., 2009; Yang et al., 2009; Rogerson et al., 2014; Hayashi-Takagi et al., 2015). Furthermore, these newly formed dentritic spines are preferentially stabilized during prolonged training and are maintained long after training is ended (Xu et al., 2009; Yang et al., 2009), suggesting a key role of synaptic structural plasticity in the formation and storage of long-term motor memories. Interestingly, given that single-pulse TMS has recently been shown to have a net suppression effect on dendritic activity of pyramidal neurons (Murphy et al., 2016), it is likely that, in the present context, TMS impaired consolidation by preventing the normal synaptic reorganization that occurs in M1 upon repeated motor exposure.

Together, our results demonstrate the causal contribution of M1 to the consolidation of motor memories when performance reaches a plateau during sensorimotor adaptation. These results suggest that repetition-dependent mechanisms within M1, possibly in conjunction with reward processing, foster the long-term storage of motor memories.

\section{References}

Bagce HF, Saleh S, Adamovich SV, Krakauer JW, Tunik E (2013) Corticospinal excitability is enhanced after visuomotor adaptation and depends on learning rather than performance or error. J Neurophysiol 109:10971106. CrossRef Medline 
Baraduc P, Lang N, Rothwell JC, Wolpert DM (2004) Consolidation of dynamic motor learning is not disrupted by rTMS of primary motor cortex. Curr Biol 14:252-256. CrossRef Medline

Bütefisch CM, Davis BC, Wise SP, Sawaki L, Kopylev L, Classen J, Cohen LG (2000) Mechanisms of use-dependent plasticity in the human motor cortex. Proc Natl Acad Sci U S A 97:3661-3665. CrossRef Medline

Bütefisch CM, Khurana V, Kopylev L, Cohen LG (2004) Enhancing encoding of a motor memory in the primary motor cortex by cortical stimulation. J Neurophysiol 91:2110-2116. CrossRef Medline

Carlton LG (1992) Visual processing time and the control of movement. In: Vision and motor control (Proteau L, Elliott D, eds), pp 3-31. Oxford: North-Holland.

Classen J, Liepert J, Wise SP, Hallett M, Cohen LG (1998) Rapid plasticity of human cortical movement representation induced by practice. J Neurophysiol 79:1117-1123. Medline

Della-Maggiore V, Villalta JI, Kovacevic N, McIntosh AR (2017) Functional evidence for memory stabilization in sensorimotor adaptation: a $24 \mathrm{~h}$ resting-state fMRI study. Cereb Cortex 27:1748-1757. CrossRef Medline

Diedrichsen J, White O, Newman D, Lally N (2010) Use-dependent and error-based learning of motor behaviors. J Neurosci 30:5159-5166. CrossRef Medline

Doyon J, Benali H (2005) Reorganization and plasticity in the adult brain during learning of motor skills. Curr Opin Neurobiol 15:161-167. CrossRef Medline

Doyon J, Bellec P, Amsel R, Penhune V, Monchi O, Carrier J, Lehéricy S, Benali H (2009) Contributions of the basal ganglia and functionally related brain structures to motor learning. Behav Brain Res 199:61-75. CrossRef Medline

Fink GR, Frackowiak RS, Pietrzyk U, Passingham RE (1997) Multiple nonprimary motor areas in the human cortex. J Neurophysiol 77:21642174. Medline

Fitts PM (1954) The information capacity of the human motor system in controlling the amplitude of movement. J Exp Psychol 47:381-391. CrossRef Medline

Fu M, Zuo Y (2011) Experience-dependent structural plasticity in the cortex. Trends Neurosci 34:177-187. CrossRef Medline

Fu M, Yu X, Lu J, Zuo Y (2012) Repetitive motor learning induces coordinated formation of clustered dendritic spines in vivo. Nature 483:92-95. CrossRef Medline

Gabitov E, Manor D, Karni A (2014) Done that: short-term repetition related modulations of motor cortex activity as a stable signature for overnight motor memory consolidation. J Cogn Neurosci 26:2716-2734. CrossRef Medline

Gabitov E, Manor D, Karni A (2015) Patterns of modulation in the activity and connectivity of motor cortex during the repeated generation of movement sequences. J Cogn Neurosci 27:736-751. CrossRef Medline

Galea JM, Vazquez A, Pasricha N, Orban de Xivry JJ, Celnik P (2011) Dissociating the roles of the cerebellum and motor cortex during adaptive learning: the motor cortex retains what the cerebellum learns. Cereb Cortex 21:1761-1770. CrossRef Medline

Galea JM, Mallia E, Rothwell J, Diedrichsen J (2015) The dissociable effects of punishment and reward on motor learning. Nat Neurosci 18:597-602. CrossRef Medline

Gerschlager W, Siebner HR, Rothwell JC (2001) Decreased corticospinal excitability after subthreshold $1 \mathrm{~Hz}$ rTMS over lateral premotor cortex. Neurology 57:449-455. CrossRef Medline

Hadipour-Niktarash A, Lee CK, Desmond JE, Shadmehr R (2007) Impairment of retention but not acquisition of a visuomotor skill through time-dependent disruption of primary motor cortex. J Neurosci 27: 13413-13419. CrossRef Medline

Hallett M (2007) Transcranial magnetic stimulation: a primer. Neuron 55: 187-199. CrossRef Medline

Hauptmann B, Reinhart E, Brandt SA, Karni A (2005) The predictive value of the leveling off of within session performance for procedural memory consolidation. Cogn Brain Res 24:181-189. CrossRef Medline

Hayashi-Takagi A, Yagishita S, Nakamura M, Shirai F, Wu YI, Loshbaugh AL, Kuhlman B, Hahn KM, Kasai H (2015) Labelling and optical erasure of synaptic memory traces in the motor cortex. Nature 525:333-338. CrossRef Medline

Herzfeld DJ, Vaswani PA, Marko MK, Shadmehr R (2014) A memory of errors in sensorimotor learning. Science 345:1349-1353. CrossRef Medline

Huang VS, Haith A, Mazzoni P, Krakauer JW (2011) Rethinking motor learning and savings in adaptation paradigms: model-free memory for successful actions combines with internal models. Neuron 70:787-801. CrossRef Medline

Kantak SS, Sullivan KJ, Fisher BE, Knowlton BJ, Winstein CJ (2010) Neural substrates of motor memory consolidation depend on practice structure. Nat Neurosci 13:923-925. CrossRef Medline

Karni A, Meyer G, Jezzard P, Adams MM, Turner R, Ungerleider LG (1995) Functional MRI evidence for adult motor cortex plasticity during motor skill learning. Nature 377:155-158. CrossRef Medline

Karni A, Meyer G, Rey-Hipolito C, Jezzard P, Adams MM, Turner R, Ungerleider LG (1998) The acquisition of skilled motor performance: fast and slow experience-driven changes in primary motor cortex. Proc Natl Acad Sci U S A 95:861-868. CrossRef Medline

Kleim JA, Hogg TM, VandenBerg PM, Cooper NR, Bruneau R, Remple M (2004) Cortical synaptogenesis and motor map reorganization occur during late, but not early, phase of motor skill learning. J Neurosci 24: 628-633. CrossRef Medline

Krakauer JW, Ghez C, Ghilardi MF (2005) Adaptation to visuomotor transformations: consolidation, interference, and forgetting. J Neurosci 25: 473-478. CrossRef Medline

Lalazar H, Vaadia E (2008) Neural basis of sensorimotor learning: modifying internal models. Curr Opin Neurobiol 18:573-581. CrossRef Medline

Landi SM, Baguear F, Della-Maggiore V (2011) One week of motor adaptation induces structural changes in primary motor cortex that predict long-term memory one year later. J Neurosci 31:11808-11813. CrossRef Medline

Ledberg A, Bressler SL, Ding M, Coppola R, Nakamura R (2007) Large-scale visuomotor integration in the cerebral cortex. Cereb Cortex 17:44-62. CrossRef Medline

Leow LA, Hammond G, de Rugy A (2014) Anodal motor cortex stimulation paired with movement repetition increases anterograde interference but not savings. Eur J Neurosci 40:3243-3252. CrossRef Medline

Leow LA, de Rugy A, Marinovic W, Riek S, Carroll TJ (2016) Savings for visuomotor adaptation require prior history of error, not prior repetition of successful actions. J Neurophysiol 116:1603-1614. CrossRef Medline

Mandelblat-Cerf Y, Novick I, Vaadia E (2011) Expressions of multiple neuronal dynamics during sensorimotor learning in the motor cortex of behaving monkeys. PLoS One 6:1-14. CrossRef Medline

Manto M, Oulad ben Taib N, Luft AR (2006) Modulation of excitability as an early change leading to structural adaptation in the motor cortex. J Neurosci Res 83:177-180. CrossRef Medline

Masamizu Y, Tanaka YR, Tanaka YH, Hira R, Ohkubo F, Kitamura K, Isomura Y, Okada T, Matsuzaki M (2014) Two distinct layer-specific dynamics of cortical ensembles during learning of a motor task. Nat Neurosci 17:987-994. CrossRef Medline

Mawase F, Uehara S, Bastian AJ, Celnik P (2017) Motor learning enhances use-dependent plasticity. J Neurosci 37:2673-2685. CrossRef Medline

McDougle SD, Bond KM, Taylor JA (2015) Explicit and implicit processes constitute the fast and slow processes of sensorimotor learning. J Neurosci 35:9568-9579. CrossRef Medline

Muellbacher W, Ziemann U, Boroojerdi B, Cohen L, Hallett M (2001) Role of the human motor cortex in rapid motor learning. Exp Brain Res 136: 431-438. CrossRef Medline

Muellbacher W, Ziemann U, Wissel J, Dang N, Kofler M, Facchini S, Boroojerdi B, Poewe W, Hallett M (2002) Early consolidation in human primary motor cortex. Nature 415:640-644. CrossRef Medline

Murphy SC, Palmer LM, Nyffeler T, Müri RM, Larkum ME (2016) Transcranial magnetic stimulation (TMS) inhibits cortical dendrites. eLife 5:e13598. CrossRef Medline

Nudo RJ, Milliken GW, Jenkins WM, Merzenich MM (1996) Use-dependent alterations of movement representations in primary motor cortex of adult squirrel monkeys. J Neurosci 16:785-807. Medline

Orban de Xivry JJ, Lefèvre P (2015) Formation of model-free motor memories during motor adaptation depends on perturbation schedule. J Neurophysiol 113:2733-2741. CrossRef Medline

Orban de Xivry JJ, Criscimagna-Hemminger SE, Shadmehr R (2011) Contributions of the motor cortex to adaptive control of reaching depend on the perturbation schedule. Cereb Cortex 21:1475-1484. CrossRef Medline

Orban de Xivry JJ, Ahmadi-Pajouh MA, Harran MD, Salimpour Y, Shadmehr R (2013) Changes in corticospinal excitability during reach adaptation in force fields. J Neurophysiol 109:124-136. CrossRef Medline 
Overduin SA, Richardson AG, Bizzi E (2009) Cortical processing during dynamic motor adaptation. Adv Exp Med Biol 629:423-438. CrossRef Medline

Pascual-Leone A, Valls-Solé J, Wassermann EM, Hallett M (1994) Responses to rapid-rate transcranial magnetic stimulation of the human motor cortex. Brain 117:847-858. CrossRef Medline

Paz R, Boraud T, Natan C, Bergman H, Vaadia E (2003) Preparatory activity in motor cortex reflects learning of local visuomotor skills. Nat Neurosci 6:882-890. CrossRef Medline

Paz R, Natan C, Boraud T, Bergman H, Vaadia E (2005) Emerging patterns of neuronal responses in supplementary and primary motor areas during sensorimotor adaptation. J Neurosci 25:10941-10951. CrossRef Medline

Picard N, Strick PL (2001) Imaging the premotor areas. Curr Opin Neurobiol 11:663-672. CrossRef Medline

Reis J, Fischer JT, Prichard G, Weiller C, Cohen LG, Fritsch B (2015) Timebut not sleep-dependent consolidation of tDCS-enhanced visuomotor skills. Cereb Cortex 25:109-117. CrossRef Medline

Richardson AG, Overduin SA, Valero-Cabré A, Padoa-Schioppa C, PascualLeone A, Bizzi E, Press DZ (2006) Disruption of primary motor cortex before learning impairs memory of movement dynamics. J Neurosci 26: 12466-12470. CrossRef Medline

Richardson AG, Borghi T, Bizzi E (2012) Activity of the same motor cortex neurons during repeated experience with perturbed movement dynamics. J Neurophysiol 107:3144-3154. CrossRef Medline

Rogerson T, Cai DJ, Frank A, Sano Y, Shobe J, Lopez-Aranda MF, Silva AJ (2014) Synaptic tagging during memory allocation. Nat Rev Neurosci 15:157-169. CrossRef Medline

Rosenkranz K, Kacar A, Rothwell JC (2007) Differential modulation of motor cortical plasticity and excitability in early and late phases of human motor learning. J Neurosci 27:12058-12066. CrossRef Medline

Rroji O, van Kuyck K, Nuttin B, Wenderoth N (2015) Anodal tDCS over the primary motor cortex facilitates long-term memory formation reflecting use-dependent plasticity. PLoS One 10:e0127270. CrossRef Medline

Schlerf JE, Galea JM, Bastian AJ, Celnik PA (2012) Dynamic modulation of cerebellar excitability for abrupt, but not gradual, visuomotor adaptation. J Neurosci 32:11610-11617. CrossRef Medline

Schulze-Bonhage A, Cichon BM, Ferbert A (1998) Cortical representation of proximal and distal arm muscles as assessed by focal transcranial magnetic stimulation. Electromyogr Clin Neurophysiol 38:81-86. Medline

Shadmehr R, Holcomb HH (1997) Neural correlates of motor memory consolidation. Science 277:921-925. CrossRef Medline

Shadmehr R, Smith MA, Krakauer JW (2010) Error correction, sensory prediction, and adaptation in motor control. Annu Rev Neurosci 33:89-108. CrossRef Medline

Smith MA, Ghazizadeh A, Shadmehr R (2006) Interacting adaptive processes with different timescales underlie short-term motor learning. PLoS Biol 4:1035-1043. CrossRef Medline

Taylor JA, Krakauer JW, Ivry RB (2014) Explicit and implicit contributions to learning in a sensorimotor adaptation task. J Neurosci 34:3023-3032. CrossRef Medline

Trempe M, Proteau L (2010) Distinct consolidation outcomes in a visuomotor adaptation task: off-line leaning and persistent after-effect. Brain Cogn 73:135-145. CrossRef Medline

Ungerleider LG, Doyon J, Karni A (2002) Imaging brain plasticity during motor skill learning. Neurobiol Learn Mem 78:553-564. CrossRef Medline

Verstynen T, Sabes PN (2011) How each movement changes the next: an experimental and theoretical study of fast adaptive priors in reaching. J Neurosci 31:10050-10059. CrossRef Medline

Werner S, Schorn CF, Bock O, Theysohn N, Timmann D (2014) Neural correlates of adaptation to gradual and to sudden visuomotor distortions in humans. Exp Brain Res 232:1145-1156. CrossRef Medline

Wise SP, Moody SL, Blomstrom KJ, Mitz AR (1998) Changes in motor cortical activity during visuomotor adaptation. Exp Brain Res 121:285-299. CrossRef Medline

Xiao J, Padoa-Schioppa C, Bizzi E (2006) Neuronal correlates of movement dynamics in the dorsal and ventral premotor area in the monkey. Exp Brain Res 168:106-119. CrossRef Medline

Xu T, Yu X, Perlik AJ, Tobin WF, Zweig JA, Tennant K, Jones T, Zuo Y (2009) Rapid formation and selective stabilization of synapses for enduring motor memories. Nature 462:915-919. CrossRef Medline

Yang G, Pan F, Gan WB (2009) Stably maintained dendritic spines are associated with lifelong memories. Nature 462:920-924. CrossRef Medline

Yin PB, Kitazawa S (2001) Long-lasting aftereffects of prism adaptation in the monkey. Exp Brain Res 141:250-253. CrossRef Medline

Yu X, Zuo Y (2011) Spine plasticity in the motor cortex. Curr Opin Neurobiol 21:169-174. CrossRef Medline 